\title{
Physical Literacy for Children Labeled With Autism Spectrum Disorder: Mothers' Experiences of Ableism, Exclusion, and Trauma
}

\author{
Kyle Pushkarenko \\ Memorial University of Newfoundland and University of Alberta \\ Janice Causgrove Dunn and Donna L. Goodwin \\ University of Alberta
}

\begin{abstract}
Countering the declining physical activity patterns of children labeled with autism spectrum disorder (ASD) has gained considerable research attention given its impact on health and quality of life. The purpose of this study was to explore how parents of children labeled with ASD understand the concept of physical literacy, based on their children's participation in community-based physical activity programs. Using interpretive phenomenological analysis, six mothers of children labeled with ASD participated in one-on-one semistructured interviews. The conceptual framework of ecological systems theory supported the rationale for the study purpose, provided structure for the interview guide, and offered a reflexive context for interpretation. Four themes were generated from the thematic analysis: From embodied movement to normative skill expectations, Be flexible, not rigid, Systematic exclusion, and Valuable? ... Absolutely! Despite experiences of marginalization, exclusion, and trauma within physical activity programs, mothers valued physical literacy development for their children given the positive outcomes of increasing family connections, engagement with peers, and enhanced wellness.
\end{abstract}

Keywords: ecological systems theory, developmental impairment, inclusive practice, interpretive phenomenological analysis, community-based programming

Autism spectrum disorder (ASD) has been described as a developmental impairment represented through variable levels of ability in such areas as communication and language, social interaction, and behavior (Centers for Disease Control and Prevention, 2016). ASD has traditionally been positioned within a

Pushkarenko is currently with Memorial University of Newfoundland, St. John's, NL, Canada. Causgrove Dunn and Goodwin are with the University of Alberta, Edmonton, AB, Canada. Pushkarenko (kpushkarenko@mun.ca) is corresponding author. 
deficit-based framework, where the uneven development of abilities is perceived as dysfunctional and/or problematic (Dinishak, 2016; Lester et al., 2014), often resulting in stigmatization and social exclusion (Buchanan et al., 2017; Must et al., 2015; Obrusnikova \& Miccinello, 2012). Researchers have also indicated that children labeled with $\mathrm{ASD}^{1}$ are susceptible to physical activity decline due to their considerable difficulty engaging in physical activity where social skills, communication, and motor abilities are essential for prolonged participation (Ayvazoglu et al., 2015; Bandini et al., 2013; Gregor et al., 2018; Pitetti et al., 2007). This susceptibility places them at increased risk for chronic disease and poorer overall quality of life (Criado et al., 2017; Curtin et al., 2014; MacDonald et al., 2011).

Over the past decade, interest in countering the declining physical activity patterns of children labeled with ASD has gained considerable research attention (Lee \& Hodge, 2017; Sowa \& Meulenbroek, 2012; Srinivasan et al., 2014). Researchers have suggested that preventing this decline may be an easily attainable goal, reversing already low levels of activity engagement (Pitetti et al., 2007). As a potential avenue for prevention, physical literacy (PL) development has been emphasized through quality sport and recreation programs, and physical education curriculum delivery for all children, including those labeled with ASD (Edwards et al., 2017; Higgs, 2010; Jurbala, 2015; Yi et al., 2020). In these contexts, PL development has been considered a means by which to address the complexities associated with reduced physical activity in children labeled with ASD, including reasons related to low levels of motivation (Obrusnikova \& Miccinello, 2012; Stanish et al., 2015), low-perceived motor skill competence (Loprinzi, Davis, \& $\mathrm{Fu}, 2015$ ), a lack of enjoyment in physical activity (Eversole et al., 2016), and impaired social and communicative abilities (Ostfeld-Etzion et al., 2016; Van Damme et al., 2015).

PL is the opportunity to capitalize on our human embodied capacity toward movement wherein "the individual has the motivation, confidence, physical competence, knowledge, and understanding to value and take responsibility for engaging in physical activities for life" (Whitehead, 2019, p. 8). According to Whitehead $(2010,2019)$, the nurturing of a positive attitude toward movement potential, and the motivation to establish a personal connection and interaction with the surrounding environment, increases opportunities for the development of physical competence. This, in turn, provides motivation for ongoing engagement; the cycle between motivations, interaction with the surrounding environment, and competence development is continuous (Whitehead, 2010, 2019).

In Canada, PL development has rapidly been adopted as a core programming priority by many organizations, including those providing services to children labeled with ASD (Tremblay et al., 2018). However, at the practitioner level, the concept of PL is not well understood, and its application has been interpreted to reflect normativity via the acquisition of fundamental movement skills as prerequisites to more advanced forms of movement (Higgs et al., 2019; Pushkarenko et al., 2020; Standal, 2015). For those with unique embodiments (i.e., individuals experiencing disability) who may not be able to achieve normatively based movement skills, PL as implemented in practice can be experienced as exclusionary and ableist (Goodwin, 2016)..$^{2}$ Although PL is purported to be an individual responsibility and inclusive of all bodies and minds, irrespective of culture, age, or ability (Whitehead, 2010, 2019), there has been little engagement with exclusionary sociocultural and political forces 
embedded in physical activity and sport for children and youth with impairments (e.g., promotion of certain types of bodies; disabling architecture and social access; hierarchical domain of instructors, coaches, and administrators; program fees; transportation; and staff competencies, Goodwin \& Peers, 2011). Rather, individuals (and their families) assume the responsibility of navigating ableist policies and practices while the structural impediments to PL development often go overlooked (Boyd, \& Goodwin, 2019; McMahon et al., 2019).

Consistent with the criticism of PL programming being exclusionary (Goodwin, 2016), Pushkarenko et al. (2020) indicate that while there is recent evidence of person-centered approaches to programming, practices implemented to promote physical activity engagement have largely emphasized the identification and correction of movement deficits and deficiencies to increase the capacity of those experiencing disability to engage in normative activities. These practices appear to contradict the conceptualization of PL as a journey occurring in the context of one's personal endowment (Whitehead, 2010, 2019), and instead portray PL as "one-size fits all" (Lloyd, 2016). If PL development occurs according to one's unique embodied potential (Jurbala, 2015, Vickerman \& DePauw, 2010), then different life experiences and capabilities of individuals living with disability must be a key consideration for program development and implementation.

Parental support is among the most important determinants of participation for children experiencing disability as they often rely on their parents for the planning and overall facilitation of their physical activity experiences (Martin \& Choi, 2009; Siebert et al., 2017). Parents are a vital part of a child's immediate environment and their roles across environmental contexts (e.g., role model, advocate, facilitator, teacher, participant) are the most influential with respect to a child's overall development and well-being (Pfeiffer et al., 2017). Therefore, it comes as no surprise to see that researchers have remarked on the importance of parents as key collaborators in the development of PL for their children (Whitehead \& Murdoch, 2006; Whitehead, 2013). As the development of PL for all children is collaboratively facilitated by parents, research accentuating parent perspectives is imperative to maintaining a commitment to responsive, respectful, and ethical PL practice (Goodwin \& Rossow-Kimball, 2012). The purpose of this study was to explore how parents of children labeled with ASD understand the concept of PL, based on their children's participation in community-based physical activity programs.

\section{Conceptual Framework-Ecological Systems Theory}

According to Pfeiffer et al. (2017), a primary factor influencing participation in physical activity by children labeled with ASD, and by association PL development, is the fit between their subjectivities and the surrounding environment. This has been labeled by Rudd et al. (2020) as "constraints-induced synergy" (p. 3), whereby affordances to move emerge as a result of the synergistic convergence of constraints related to the task, individual, and environment. Movement is considered to be a complex, dynamic, and reciprocal interplay of one's interactive experiences with the physical and social world and as such, it is influenced by exposure to various environmental (e.g., built environment) and social influences 
(e.g., parents, peers, teachers; O’Sullivan et al., 2020; Rudd et al., 2020). Given the influential role of parents as advocates and facilitators of their child's development and physical activity involvement (Martin \& Choi, 2009; Pfeiffer et al., 2017; Siebert et al., 2017), it is important to understand their perspectives. Applied to a parent's understanding of the concept of PL, ecological systems theory (EST) can provide valuable information about the interactive layers of social and environmental influence in the development of PL, and to which their children are exposed in community-based physical activity programs (Bronfenbrenner, 1979, 1992).

EST consists of a network of five overlapping and bidirectional environmental influences that mediate organismic-environmental interaction: microsystem, mesosystem, exosystem, macrosystem, and chronosystem (Bronfenbrenner, 1979, 1992). The microsystem consists of influences from sources that are immediate and direct, such as parents and physical activity instructors that afford participation opportunities (Gabbard \& Krebs, 2012; Huit, 2012). The mesosystem contains the influence occurring as a result of the interaction between the microsystems (e.g., parent-instructor interactions, the constructed physical environment context). The exosystem represents the larger social system in which an individual is not an active participant (Rosa \& Tudge, 2013). In other words, the exosystem involves positive or negative influences from a social structure that there is no direct involvement in (e.g., workplace attitudes and culture of a physical activity organization and its staff; Berk, 2013). The macrosystem can be thought of as a societal blueprint comprised of cultural values, subculture interests, or other broad contexts (Bronfenbrenner, 1992), with reference to belief systems, societal values, cultural customs and principles, and lifestyle behaviors (Berk, 2013; Rosa \& Tudge, 2013). Macrosystem influences are diverse and can include those at an organizational level (e.g., policies, procedures, and program structure of a physical activity organization) to a broader societal level (e.g., the value placed on standardization and normative skill development). Finally, the chronosystem encompasses the dimension of time, comprised of major life transitions (e.g., maturation) and environmental events that transpire over the course of development, including organismic (e.g., illness, injury) and sociohistorical events (e.g., implementation of physical activity restrictions for health-related reasons; Rosa \& Tudge, 2013).

\section{Methodology and Method}

As our aim was to interpret subjective experiences, this study was grounded within an interpretivist research paradigm, comprised of a relativist ontology, a transactional/subjective epistemology, and a hermeneutic and dialectical methodology (Kivunja \& Kuyini, 2017; Lincoln et al., 2011). We assume that multiple forms of social and experiential realities exist, that knowledge is subjective, and that an interactive and interpretive process between researcher and participant subjectivities cocreates knowledge. We acknowledge our varied roles, ethnicities, positions, and identities are embedded in the processes and outcome of research (Milner, 2007). The authors are experienced qualitative researchers, parents, White, and nondisabled, with backgrounds in professional practice and teaching adapted physical activity. 
Consistent with the paradigmatic assumptions, interpretative phenomenological analysis (IPA) was the research approach used, providing a systematic means by which to explore and understand the lived experience of others (Smith, 2017). Grounded in phenomenology, hermeneutics, and idiography, IPA enables the researcher to delve into how people make sense of their personal and social worlds (Shinebourne, 2011; Smith et al., 2009). Phenomenology is concerned with the study of core structures of subjective experience. The aim is to identify the essential components of a phenomenon (i.e., PL) that makes it exclusive or distinctive from others (Markula \& Silk, 2011). Hermeneutics is the study of interpretation and meaning (Shinebourne, 2011); the researcher attempts to understand the subjective experiences of the participant and make meaning based upon this understanding (Markula \& Silk, 2011; Smith et al., 2009). Idiography is an in-depth focus on the particular. Within IPA research, one participant's experiences are analyzed in-depth before going on to the next, to value each participant's diverse and variable experiences (Eatough \& Smith, 2017). Finally, a more general cross-participant analysis aimed at uncovering convergence and divergence across cases occurs (Smith et al., 2009).

Following approval from the University's Institutional Ethics Review Board, community organizations known to have physical activity programs addressing the specific needs of individuals labeled with ASD and incorporating the principles of PL into their program structure received a recruitment letter outlining the study's specifics. Representatives from each organization sent the recruitment letters to prospective participants, who then contacted the principal investigator expressing interest in the study. Once vetted according to the eligibility criteria, the principal investigator invited those who met the criteria to take part in the research. Prior to the commencement of data collection, the principal investigator provided additional information pertaining to the participant's involvement in the study (e.g., benefits, risks, time commitment, and study withdrawal) and each participant provided written informed consent. To protect anonymity, all identifying information was removed from research materials, and pseudonyms were assigned to each of the participants.

\section{Participants}

Consistent with IPA, a purposive and homogeneous sample participated in the study. Six parents met the eligibility criteria, which included having a child: (a) with a label of ASD (as per parental report), (b) who was between the ages of 7 and 12 years, and (c) who had been enrolled in community-based physical activity programs emphasizing the development of PL within the previous 12 months. Parents of children of this age were targeted, as this is when children are most susceptible to physical activity decline (Gregor et al., 2018; Must et al., 2015). Program enrollment within the previous 12 months was chosen to optimize accurate recollection of experiences. Four participants were recruited from one organization located in a large Canadian city, and the remaining two via snowball sampling (Patton, 2015).

Although not stipulated during recruitment, all six parents were mothers whose average age was 43 years (range $30-53$ years). The average age of their 
children with a label of ASD was 8.2 years (range 7-10 years). There were five two-parent families; four were single-child families (two were three-child families). All participants were White, had postsecondary education, and four worked outside of the home full time. The mothers reported that their children had participated in adapted physical activity programs for an average of 3.8 years (range 1-8 years).

\section{Community Programs}

The community programs in which the participants' children took part emphasized PL via play and/or the acquisition of motor skills: attributes reflective of the Active Start and FUNdamentals stages of the Canadian Sport for Life framework (Higgs et al., 2019). Over periods of 8 and 12 weeks at various times of the year, the children took part in multiple program activities (both as individuals and within groups) from once to several times per week. With few exceptions (e.g., soccer and swimming programs), the children participated in specialized, segregated programs specifically designed for individuals experiencing disability. Physical activity practitioners who had completed a PL instructor program or possessed training in activity-specific motor skill development led the programs. Parents were present during the programs and available if support of their children was necessary; however, their roles were primarily that of being an observer.

\section{Data Collection}

The aim of data collection in IPA inquiries is to obtain detailed first-hand accounts of experiences. To elicit stories, thoughts, and feelings, the participants took part in face-to-face, audio-recorded, semistructured interviews (Smith et al., 2009). The first author conducted all interviews, at a location and time convenient to the participants. An interview guide informed by the research purpose and conceptual framework was used to encourage participants to express their feelings, knowledge, and thoughts about PL. Descriptive (e.g., What does PL mean to you? Through your previous experiences, what has your role been in facilitating your child's PL development?), narrative (e.g., Can you tell me how you arrived at your understanding of PL? In your experience, what has been the best means of facilitating PL development for your child?), and structural questions (e.g., How do things like knowledge and understanding, motivation and confidence, and/or personal responsibility fit in with your understanding of PL for your child?) comprised the interview guide. Two experienced qualitative researchers reviewed the guide prior to the first interview. Revisions were made based on their feedback related to the integration of the conceptual and methodological frameworks (Sparkes \& Smith, 2014).

The interviews averaged $62 \mathrm{~min}$ in duration (ranging from 50 to $75 \mathrm{~min}$ ). Follow-up interviews of approximately 15 min took place with two participants, to clarify information provided during their initial interviews, 6 and 12 weeks after the initial interviews. All interviews were transcribed verbatim, after which participants were invited to review their own transcripts and provide clarification through the addition of information (Smith \& McGannon, 2018; Tracy, 2010). Five of the six mothers responded, and no additions were required. 
In addition to the interviews themselves, the first author recorded field notes during and after the interviews. Brief comments noted during the interviews were elaborated shortly afterward, expanding on what the participants discussed, thoughts about the conversation, and considerations for analysis. Reflexive notes that included topics such as the researcher's positionality, research decisions made and their rationale, and thoughts about the power dynamics between the participants and the researcher, were also recorded (Merriam et al., 2001). The field notes became points of reflexion during the analysis and interpretation of the findings.

\section{Data Analysis}

An inductive, line-by-line analysis of the interview transcripts was completed by the first author using Smith et al.'s (2009) six-step framework as a guide. The second and third authors acted as "critical companions" (Paterson \& Higgs, 2005, p. 340) by engaging in discussions with the first author, and providing critical appraisal and feedback regarding reflexivity, interpretations, and the presentation of findings (Paterson \& Higgs, 2005; Smith \& McGannon, 2018). The analysis proceeded with the first author becoming fully immersed in the data, listening to the audio recordings of the first participant's interview, and reading and rereading the corresponding transcript. Next, initial idiographic experiences for that participant were noted by adding descriptive, linguistic, and conceptual notes about word choice, tone of voice, and recalled body language (Smith et al., 2009). These notes were used to develop preliminary themes, and then connections were sought across the preliminary themes, capturing each participant's account. Here, lists of preliminary themes were printed out, cut up, and then physically manipulated to determine relationships to each other. Once this process was completed for the first participant, it was repeated with each of the other participants. The final step of the analysis was to move between individual voices, and group patterns, to identify the most important themes of the collective group. The field notes supplemented the interview data, providing the researchers context to conceptually return to the interview setting during analysis (Mayan, 2009; Phillippi \& Lauderdale, 2018). The developed themes were shared with and discussed among the authorship team until consensus was reached.

The conceptual framework of EST (Bronfenbrenner, 1979, 1992) was drawn upon to bring a deeper understanding of the meanings held by each participant, and the collective group (Collins \& Stockton, 2018). A summary of the preliminary themes was sent to the respective participants via e-mail, serving as an invitation to explore gaps and share common interpretations (i.e., member reflexions; Smith \& McGannon, 2018). All six of the participants accepted the invitation. Small word changes were incorporated into the idiographic summaries and reflected on deeply in the presentation of the final themes.

\section{Research Quality}

The quality of the research was addressed according to four criteria identified for IPA: (a) sensitivity to context, (b) commitment and rigor, (c) transparency and coherence, and (d) impact and importance (Smith et al., 2009; Yardley, 2000). 
The authors achieved sensitivity to context by demonstrating a theoretical, philosophical, and methodological awareness of the research process, including the relevant literature on PL, previous work using EST as a conceptual framework, and IPA as a research approach. Additionally, sensitivity to context was demonstrated through the use of a purposeful and homogenous sampling strategy, ongoing researcher reflexion, highlighting contextual meanings through thick description, and being sensitive to each participant's experiences in turn, thus adhering to the idiographic nature of IPA research (Smith et al., 2009, Yardley, 2000; Zitomer \& Goodwin, 2014).

The authors established commitment and rigor by developing thorough data collection and analysis strategies, by providing participants the opportunity to reflect on their interview transcripts and thematic trends, and by comparing participant themes against the reflexive interview notes, thus adding a level of credibility to the research (Zitomer \& Goodwin, 2014). The authors achieved transparency and coherence by providing a detailed account of the research methodology and research approach, and full disclosure of the participant's role in the research prior to their involvement in the study. Theory was used throughout the research process (e.g., analysis and interpretation, and discussion context), further strengthening the cohesiveness and consistency of the study. Impact and importance are determined by what the reader considers as important and what they do with results of the study. By offering a detailed description of the research process, readers can decide whether the findings are pertinent to them and transfer them to their context.

\section{Findings}

Four themes were generated from the analysis. The themes represent the mothers' understanding of the concept of PL that is contrary to its foundation as inclusive of all bodies and minds irrespective of culture, age, or ability. The mothers came to understand PL as a concept that promoted normative skill development, was inflexible in performance expectations, and systematically excluded their children. Even so, the parents understood that achieving a form of PL held value and importance. The themes are (a) From embodied movement to normative skill expectations, (b) Be flexible, not rigid, (c) Systematic exclusion, and (d) Valuable? ... Absolutely!

\section{From Embodied Movement to Normative Skill Expectations}

Participants' experiences revealed that understanding PL derived from diverse sources including online postings, brochures, direct communication with physical activity instructors, and observing their child. Knowledge of what PL is ranged from simply engaging in movement and the embodied sensations that result from this movement to specific normative-based skill expectations. Beyond understandings of "what" PL is, participants also believed that they shouldered the majority of the responsibility for their child's PL development (i.e., the "how"), often having to engage in negotiations between the needs of their children and the expectations of the physical activity environment. Extending this, participants 
considered that their roles and responsibilities were more extensive than those of other parents. As Maya stated,

We (the parents) are responsible for getting him (her son) engaged . . . . I don't get to just sign him up for something and show up the first day. It's, I call ahead of time, I see if I can come down to take pictures of where it is at so I can make a social story so he knows where he is going to be.

Other forms of parental involvement included participating alongside their children and mentoring instructors.

After acknowledging that her exposure to PL was restricted to literature posted through an online support group and therefore was rather limited, Shirley equated PL to one's level of physical awareness, or "knowing where their body is in space." She elaborated by stating that conversations with others about PL were nonexistent: "I don't have anyone in my own personal life that talks to me about physical literacy." Theresa learned from one of her son's physical activity instructors that PL is related to enhanced motor skill development and "it's like being able to move and perform movements more accurately." Maya derived her understanding from a flyer that also aligned PL with motor skill development. However, she would have appreciated a more in-depth conversation about the meaning of PL, "because once a parent is made aware of it, they may try to encourage it more." Relatedly, Donna stated that "parents have to take ownership" for ensuring their child's success and understood PL according to developmental performance standards:

My understanding of it is basically the ability to move through the movements at the level that you should be able to move through the movements at. It's like being able to perform physical activity in a way that it is appropriate to where you should be able to perform physical activity.

Donna further expressed that taking ownership for her child's development was difficult as PL strategies were elusive, "No one is actually translating that information to the people who can make a difference. It has to translate and trickle down to me."

Monica's understanding derived from interactions with her son who required physical assistance in movement activities. Her understanding aligned with the notions of embodiment and connectedness with one's surroundings, rather than facilitating normalized development yet still required her direct involvement in the program:

It's just any movement ... physical movement that connects a person to either a specific activity, or umm ... . or the earth. I mean to me, and of course, this is all shaped based on my son, umm ... crawling through the grass and him being able to feel the grass underneath his hands, and what that feels like and what it is. And maybe, feeling moist dirt ... that's all providing him experience and like connectedness to his surroundings ... it's not just the actual physical activity itself-it's all those other little steps and things that he touches, sees, smells ... those are learning opportunities for him ... so I guess it's just learning about kind of the whole world around him, and what these things all are. 
Susan echoed Monica's understanding stating, "For one child one thing may be important, but for another child with autism it might be totally different ... each kid will kind of have their own thing." Susan acknowledged the larger, and even managerial role she played in her son's participation that included being able "to break down where he's at for these people trying to work with him so that they know how to work with him ... it's a huge role." In summary, PL meant different things to the participants and was based on their interaction with various influences and sources of information in the immediate environment (e.g., microsystem of parent self-knowledge, child's instructors) and connections with neighborhood facilities and peers (e.g., mesosystem of online presence, print materials, program facilities).

\section{Flexibility, Not Rigidity}

Observing the diversity of their children's abilities, interests and needs, and how they interacted with surrounding environments, participants collectively understood that flexible, individualized programs were essential for PL development. Moreover, they believed it was more important to attend to the needs of their child than adhere to rigidly planned PL activities and structure.

Declaring her displeasure for PL programs, Maya suggested that instructional approaches that lacked individualization devalued her son's strengths and capabilities. She felt these approaches contributed to him not feeling like he belonged, leaving him deflated and less likely to want to return. "I think the older he gets, the more negative it does get for him with physical activity ... the older he gets and the more his gap grows especially in the ability to keep up."

Similarly, Theresa expressed frustration with programs that held to rigid performance standards and lacked attention to the needs of each child (e.g., instruction, types of activities) believing her son "can do a lot of things, but not those things." From Theresa's perspective, a normatively based approach accentuates and puts on display what cannot be done, rather than highlighting what can be done. As summarized succinctly by Donna, "children who are disabled clearly cannot do physical activity the same way ... . Physical activity, first and foremost, should be enjoyable. Because if it is not, it won't be sustained."

As a result of previous experiences in the physical activity setting, the participants sought a physically and socially supportive environment for their children. For her son, Maya felt that increased amounts of activity, numbers of people, and noise negatively impacted his ability to engage in physical activity:

A lot of the times it is the busyness of it all, like when we were in soccer there's eight different teams playing on four fields and kids are everywhere and that is hard for him to navigate so usually finding programs that are smaller, quieter, less busy, less hectic. That works.

Monica reinforced the importance of the availability of flexible spaces to promote success: "They're [the staff] really great with him, even from the beginning ... letting us go to this kind of quieter area when he needs a break." The parents' experiences again reflected the overlapping influences of the microsystem and mesosystems. The participants appreciated flexibility in instructors' performance expectations that emphasized fun for their children (microsystem) and 
the physical environment in which they participated (mesosystem). Instructors (as part of the microsystem) who had direct contact with the children and did not individualize programs but instead emphasized performance-based PL (normative skill-based instruction that accentuated differences) were deemed to be disrespectful of the children and in turn, their families.

\section{Systematic Exclusion}

The experiences of participants highlighted a collective understanding of PL as an exclusive concept. Despite claims of inclusivity, the mothers encountered programs emphasizing practices that were not necessarily welcoming of children experiencing disability. They indicated that opportunities for their children to develop PL were few and far between, and when provided, both their children and their participation were marginalized. Maya explained that she encountered resistance and limited support when simply inquiring about a program for her son: "It's like when you just call and say my child has autism they're automatically like 'you know I don't think this is a good fit for him." Essentially, exclusion is based on the label of ASD and workplace norms about who is permitted into physical activity programs (e.g., social influences of an exosystem that values the well-behaved, normally developing, independent, and compliant). Susan described her son's involvement in an activity program in which his enrollment was permitted, but where the instructors dismissed him from instruction as though he was not worth the effort, allowing him to do "whatever he wants to do" and reflecting a workplace culture that perpetuates systemic exclusion.

Monica highlighted an instructor's reluctance, and even resistance, to incorporate parents' recommendations into their instructional practice. She highlighted her disappointment and frustration with an instructor who was not receptive to her input, rejecting her as a knowledge holder, thereby creating an unnecessary and dismissive power dynamic:

You know one thing I have learned is that every parent to a typical developing child or to a non-typically developing child, you are your child's best expert. You know your kid better than anyone else I believe, and she [the instructor] never was very receptive to any suggestions or observations that I would make.

Monica also expressed frustration about the level of instructor support within her son's programs, recounting how normative expectations held by the instructor led to the depreciation of her son's accomplishments, even though the program was designed to celebrate individual milestones:

Rather than just giving him that praise she said, "[Name], finally you've noticed something." I thought, well great ... thanks for that ... she pissed me off. I just thought, you know, how about we just celebrate him and what he did, rather she just focused on "all year you've been a bad boy ... you didn't, you know, meet the expectations."

Participants expressed how negative attitudes and an overall false sense of support were offered as initial welcoming attitudes by those within the activity environment quickly deteriorated when desired outcomes were not being achieved. 
In such situations, inclusion was advocated for and supported, yet only if the aesthetic of the programs was not disrupted and normative expectations could be upheld. Theresa referred to her son's participation in a program where instructors endorsed inclusivity, but did not necessarily fully practice it:

He was in [program name] and he loved it ... . But, as it got more serious, [instructors] were like, "This is not going to work out" ... so, everyone says that they want to be supportive until something isn't perfect.

Questioning the support of her child in more structured sport programs, Maya encountered negativity on behalf of other parents whose children (not experiencing disability) were also participating. She stated that "other parents don't want your child to be in the program because they might take their child down or their child's team might not win." These attitudes reflected a substantial barrier to future engagement in such activities. The interaction of undertrained staff, judgmental attitudes of other parents (mesosystem influences), and a workplace that perpetrates and perpetuates exclusion and humiliation as an acceptable norm (exosystem) leads to children and families being marginalized within and through physical activity. The concept of PL founded on participation of all bodies and minds in lifelong participation in physical activity has become, within some contexts, a place of hurt, rejection, and humiliation.

\section{Valuable? ... Absolutely!}

Despite the variable understandings of PL and the exclusion they and their children have endured, all participants believed that PL development held value for their children and their families through improved family connections, increased engagement with peers, and enhanced wellness (face-to-face interactions with others in the microsystem). According to Shirley, her son's developing PL provided "a positive bonding experience," enabling family members to participate in activities together. Similarly, Donna spoke about family fun and connection through physical activity:

We play a lot. We play a lot, and I wish I could explain to people how that has been the key to our relationship as a family unit. Like it's ... physical activity is fun, right? It's really fun to do, so we do it together all the time . . . it's the most valuable time we spend as a family.

Susan also shared how PL enhanced family time. She explained that her enjoyment was heightened simply knowing that her son could participate alongside her and her husband: “ . . . it [physical activity] is part of our life. It's like that's just what we do, so we obviously wanted to incorporate having our children doing the same thing that we do as parents." Monica valued her son's PL development because it allowed her to discover more about him. She spoke about "learning how he expresses his likes and his dislikes enables me to get to know him even better."

The participants also valued PL development because it opened up additional opportunities for their children to engage alongside peers of a similar age. Theresa mentioned that her son's comfort level increased, providing him with a level of "self-esteem" that contributed to his confidence to engage with friends. Similarly, 
Maya explained the potential for future participation with others, stating that "the value for him is that he is learning these [skills] and hopefully going to ... we're setting him up to be doing [engaging in physical activity] when we're not there."

Finally, the participants valued PL development because of its physiological and psychological benefits. For example, Donna expressed that, "the running and the jumping was serving some sort of parasympathetic purpose just because when we took that element away, we noticed major changes both physiologically and in his demeanor." Additionally, she added that physical activity contributed to her son's overall health given that he has selective eating habits.

Participants' expressions of value regarding PL development for their children highlighted bidirectional influences within the child's immediate microsystem. On one hand, participants indicated that engagement in programs emphasizing PL development benefitted their children directly (e.g., enhanced well-being and social connectivity). On another, participants (as part of their child's microsystem) stated that as a result of their children benefitting, they reciprocally prospered with additional opportunities for physical activity engagement alongside their child, improved family cohesion, and an increasingly positive household environment overall.

\section{Discussion}

Applying EST to the mothers' understanding of PL illuminated the interactive layers of social, cultural, and environmental influence on its application to community physical activity programs. For these parents, PL came to mean the achievement and application of normatively based fundamental motor skills (Higgs et al., 2019; Pushkarenko et al., 2020; Standal, 2015). Outcome- and competence-based PL programs promote a corporeal standard for human movement and kind of body (the normate) based in "typical" motor development and culturally valued physical activity and sport. It is not surprising that the parents understood PL as exclusionary (Goodwin, 2016). The mothers sought programs that welcomed movement diversity but came to understand PL in practice as that which diminished their children's abilities, characterized them as abnormal (stigmatized as not a good fit), and turned them away as undesirable or unsuitable (Campbell, 2001, 2009). PL programmers are perpetuating and perpetrating stigmatization and social exclusion through a deficit-based view of ASD (Buchanan et al., 2017; Must et al., 2015; Obrusnikova \& Miccinello, 2012). PL in practice reflected a complacent acceptance of ableism as a basis for its processes and practices. PL as an opportunity to nurture positive attitudes toward movement potential was a lost promise to the mothers and their children.

Multiple influences impacted the mothers' understanding of PL. At the microsystem level of mother and children interaction, there was a genuine desire to support their children's movement development, a finding supported by others (Martin \& Choi, 2009; Siebert et al., 2017). Their lack of a uniform understanding of PL however, reflected poor communication between the mothers and the program instructors as the next level of influence, or the mesosystem level, on child development (Bronfenbrenner, 1995; Guy-Evans, 2020). Tensions emerged as parents struggled with the influence of the normate, an ableist exosystem 
influence of social beliefs, in instructors' narrow understanding of movement competency. The parents exposed an underlying ableism in how PL has been interpreted, promoted in instructor training programs, and ultimately applied to physical activity programs. The premise behind PL and the interactive influences impacting its educational practice supports a particular kind of child, one who falls along a typical trajectory of development. The ethical implications of the systemic exclusion of children in a national wide programming priority to enhance physical well-being are cause for pause (Goodwin \& Rossow-Kimball, 2012).

Good relationships shape children's development in positive ways (GuyEvans, 2020). In their absence, the mothers assumed responsibility for the meaningful engagement of their children by locating programs, preparing their children, and managing interactions with instructional staff and other parents (Martin \& Choi, 2009; Siebert et al., 2017). Furthermore, this responsibility took the form of hidden parental labor practices such as being physically present, modeling for instructors, or monitoring behavior in stressful environments (Goodwin \& Ebert, 2018). This finding is consistent with other studies describing hidden parental labor practices in other physical activity settings for individuals experiencing disability (Ayvazoglu et al., 2015; Hodge \& Runswick-Cole, 2013; Goodwin, 2017; Goodwin \& Ebert, 2018). In assuming this labor, the exclusionary aspects of PL in practice were masked as parents assume the roles not being assumed by instructors, or microsystem influencers, and the exosystem social structure of ableism. This reliance on parents to facilitate and support physical activity experiences for their children calls into question whether some PL programs are as inclusive as claimed (Whitehead, 2010, 2019).

The levels of influence on the mothers' understanding of PL are interactive, complex, and difficult to disentangle. The parents called for a flexible understanding of PL development. Yet, at the microsystem level, instructors who provided skill-based programs valued (either consciously or unconsciously) ableist views of movement and physical activity by comparing ability to a standard of achievement (Campbell, 2001, 2009). In doing so, an undesirable context for PL development was fostered, resulting in direct negative, and even traumatic social and physical spaces for the mothers and their children (e.g., verbal micro aggressions by instructor; Cairney et al., 2019; Goodley \& Runswick-Cole, 2011; Goodwin \& Eales, 2020; Hodge \& Runswick-Cole, 2013). Instructors' lack of familiarity with principles of adaptation and strategies for analyzing task components, or the interaction of the instructor with the formal training structures of the exosystem may be an underlying cause. Can instructors continue to practice beyond their formal training? If not, then where does the impetus for change lie? The blueprint of exclusion, ableism, and promotion of the normate is reflective of macrosystem cultural value system (Coates, 2011). Parents are hiding the ugly secret of exclusion at the child or family and instructor microsystem level, but the ills of interactive influences are woven throughout the system and will continue to plague PL programs until an ecological understanding of the influences families and their children endure in community level programs is recognized (Goodwin \& Peers, 2011; Guy-Evans, 2020; Spencer-Cavaliere et al., 2017).

Systematic exclusion, based in ableism, is culminated in a trauma inducing, emotionally dangerous, and even humiliating context for children labeled with ASD (Campbell, 2008; Hodge \& Runswick-Cole, 2013), and is in contrast to the 
widely accessible philosophical underpinnings of PL that promotes the inclusion of all, regardless of ability (Whitehead, 2010, 2019). Is not an embodied understanding of PL for all children needed? Has PL become another name for the development and application of based fundamental motor skills under an umbrella of typical motor development? Beyond this, what is the contribution of PL, except to promote the normate and reject and marginalize other? Nevertheless, the resilience of parents never ceases to surprise. Through rejection, marginalization, traumatization, and expended hidden labor, the mothers valued physical activity and looked to PL programs to support them in this family value (Bremer et al., 2016; Lang et al., 2010; Sowa \& Meulenbroek, 2012). How sad, however, to endure negative interactions because something is better than nothing.

\section{Limitations}

We acknowledge several limitations to the study. Mothers of children labeled with ASD comprised the sample of participants in the study, and therefore the findings may not be transferable to other parents. However, naturalistic generalization and transferability of the findings may occur for the reader if the experiences of the mothers of this study resonate with readers' personal experiences of PL or bring meaning to the experiences of mothers of children with differing abilities (Smith, 2018). As well, mothers were asked to retrospectively recall their experiences over a 12-month period. Despite the authors' beliefs that this time period was optimal for the recall of experiences, "access to experience is always dependent on what participants tell us about that experience" (Smith et al., 2009, p. 3). Memories are culture creations represented by language and their meanings are "embedded in the contexts of explanatory and interpretive discourse" (Brockmeier, 2015, p. 2). We hold that the participants, as knowledge holders of their lived experiences, shared their storied lives in ways that enabled self-knowledge and the social and cultural interpretations of that which they deemed memorable. Finally, the authors of the study, while all parents, are not parents to children experiencing disability, bringing a nondisability perspective to the analysis, interpretation, and dissemination of the participants' stories.

\section{Conclusions and Future Directions}

The mothers' understanding of PL as inaccessible to their children is consistent with Goodwin's (2016) assertion that exclusion is the product of discrepancies between conceptualizations of PL and the practices that facilitate its development. Given repeated traumatization, marginalization, and exclusion, these mothers may question the labor extended and the value of PL programs; is the potential harm worth the benefits (Goodwin \& Eales, 2020)? The ecological view taken in this study illuminated the extent of the work to be done. Further research into the inclusiveness of the construct of PL is needed from physical, pedagogical, and policy perspectives, providing insight into the dynamics associated with individual-environmental interactions in PL development for individuals experiencing disability over the lifespan. There is also much to be learned from bringing an embodiment or ableism lens to the ways in which PL is experienced by children. 


\section{Acknowledgments}

The authors express their sincere gratitude to the parents for sharing their stories, without which this study would not have been possible. They also acknowledge the financial support of the University of Alberta's Faculty of Graduate Studies and Research, Social Sciences and Humanities Research Council of Canada, Autism Research Council of Edmonton, and Autism Edmonton.

\section{Notes}

1. The phrase "labeled with ASD" was used in this paper to align ASD to a social model of disability, where disability and impairment are not synonymous, and impairment does not automatically equate to nonnormative biological function (Peers et al., 2014).

2. Ableism refers to "a network of beliefs, processes and practices that produces a particular kind of self and body (the corporeal standard) that is projected as the perfect, species-typical and therefore essential and fully human" (Campbell, 2001, p. 44). Disability then, is a diminished state of being human characterized as less able, abnormal, and undesirable (Campbell, 2001, 2009; Goodley \& Runswick-Cole, 2011; Hodge \& Runswick-Cole, 2013).

\section{References}

Ayvazoglu, N.R., Kozub, F.M., Butera, G., \& Murray, M.J. (2015). Determinants and challenges in physical activity participation in families with children with high functioning autism spectrum disorders from a family systems perspective. Research in Developmental Disabilities, 47, 93-105. PubMed ID: 26368652 https://doi.org/10. 1016/j.ridd.2015.08.015

Bandini, L.G., Gleason, J., Curtin, C., Lividini, K., Anderson, S.E., Cermak, S.A., ... Must, A. (2013). Comparison of physical activity between children with autism spectrum disorders and typically developing children. Autism: The International Journal of Research and Practice, 17(1), 44-54. https://doi.org/10.1177/13623613 12437416

Berk, L.E. (2013). Child development (9th ed.). Pearson Education.

Boyd, K., \& Goodwin, D.L. (2019). "It's hard when people try and get their kids away from Cole": A family's experiences of (in)dignity in leisure settings. Adapted Physical Activity Quarterly, 36(2), 223-241. https://doi.org/10.1123/apaq.2018-0034

Bremer, C., Crozier, M., \& Lloyd, M. (2016). A systematic review of the behavioural outcomes following exercise interventions for children and youth with autism spectrum disorder. Autism, 20(8), 899-915. PubMed ID: 26823546 https://doi.org/10.1177/ 1362361315616002

Brockmeier, J. (2015). Beyond the archive: Memory, narrative, and the autobiographical process. Oxford University Press.

Bronfenbrenner, U. (1979). The ecology of human development: Experiments by nature and design. Harvard University Press.

Bronfenbrenner, U. (1992). Ecological systems theory. In R. Vasta (Ed.), Six theories of child development: Revised formulations and current issues (pp. 187-249). Jessica Kingsley.

Bronfenbrenner, U. (1995). Developmental ecology through space and time: A future perspective. In P. Moen, G.H. Elder, Jr., \& K. Lüscher (Eds.), Examining lives in context: Perspectives on the ecology of human development (pp. 619-647). American Psychological Association. 
Buchanan, A.M., Midiema, B., \& Frey, G.C. (2017). Parents' perspectives of physical activity in their adult children with autism spectrum disorder: A social-ecological approach. Adapted Physical Activity Quarterly, 34(4), 401-420. PubMed ID: 28985096 https://doi.org/10.1123/apaq.2016-0099

Cairney, J., Dudley, D., Kwan, M., Bulten, R., \& Kriellaars, D. (2019). Physical literacy, physical activity and health: Toward an evidence-informed conceptual model. Sports Medicine, 49(3), 371-383. PubMed ID: 30747375 https://doi.org/10.1007/s40279019-01063-3

Campbell, F. (2001). Inciting legal fictions: Disability's date with ontology and the ableist body of the law. Griffith Law Review, 10, 42-62. https://core.ac.uk/download/pdf/ 143871776.pdf

Campbell, F.K. (2008). Exploring internalized ableism using critical race theory. Disability \& Society, 23(2), 151-162. https://doi.org/10.1080/09687590701841190

Campbell, F.K. (2009). Contours of ableism: The production of disability and ableness. Palgrave MacMillan.

Centers for Disease Control and Prevention. (2016). Developmental disabilities. http:// www.cdc.gov/ncbddd/developmentaldisabilities/facts.html

Coates, J. (2011). Physically fit or physically literate? How children with special educational needs understand physical education. European Physical Education Review, 17(2), 167-181. https://doi.org/10.1177/1356336X11413183

Collins, C.S., \& Stockton, M.C. (2018). The central role of theory in qualitative research. International Journal of Qualitative Research, 17(1), 1-10. https://doi.org/10.1177/ 1609406918797475

Criado, K.K., Sharp, W.G., McCracken, C.E., Dong, L., Scahill, L., De Vinck-Baroody, O., ... Scahill, L. (2017). Overweight and obese status in children with autism spectrum disorder and disruptive behavior. Autism, 22(4), 450-459. PubMed ID: $28325061 \mathrm{https}: / /$ doi.org/10.1177/1362361316683888

Curtin, C., Jojic, M., \& Bandini, L.G. (2014). Obesity in children with autism spectrum disorder. Harvard Review of Psychiatry, 22(2), 93-103. PubMed ID: 24614764 https:// doi.org/10.1097/HRP.0000000000000031

Dinishak, J. (2016). The deficit view and its critics. Disability Studies Quarterly, 36(4). https://doi.org/10.18061/dsq.v36i4.5236

Eatough, V., \& Smith, J.A. (2017). Interpretative phenomenological analysis. In C. Willig \& W. Stanton-Rogers (Eds.), The SAGE handbook of qualitative research in psychology (2nd ed., pp. 193-211). Sage.

Edwards, L., Bryant, A., Keegan, R., Morgan, K., \& Jones, A. (2017). Definitions, foundations and associations of physical literacy: A systematic review. Sports Medicine, 47(1), 113-126. PubMed ID: 27365029 https://doi.org/10.1007/s40279-016-0560-7

Eversole, M., Collins, D.M., Karmarkar, A., Colton, L., Quinn, J.P., Karsbaek, R., .. . Hilton, C.L. (2016). Leisure activity enjoyment of children with autism spectrum disorders. Journal of Autism and Developmental Disorders, 46(1), 10-20. PubMed ID: 26210514 https://doi.org/10.1007/s10803-015-2529-z

Gabbard, C., \& Krebs, R. (2012). Studying environmental influence on motor development in children. Physical Educator, 69(2), 136-149. https://search-proquest-com.qe2aproxy.mun.ca/scholarly-journals/studying-environmental-influence-on-motor/docview/ $1372483819 /$ se-2?accountid $=12378$

Goodley, D., \& Runswick-Cole, K. (2011). The violence of disablism. Sociology of Health \& Illness, 33(4), 602-617. PubMed ID: 21226732 https://doi.org/10.1111/j.14679566.2010.01302.x

Goodwin, D., \& Ebert, A. (2018). Physical activity for disabled youth: Hidden parental labor. Adapted Physical Activity Quarterly, 35(4), 342-360. PubMed ID: 30336688 https://doi.org/10.1123/apaq.2017-0110 
Goodwin, D., \& Peers, D. (2011). Disability, sport and inclusion. In S. Dagkas \& K. Armour (Eds.), Inclusion and exclusion through youth sport (pp. 186-202). Routledge.

Goodwin, D.L. (2016). Youth sport and dis/ability. In K. Green (Ed.), Routledge handbook of youth sport (pp. 308-320). Routledge.

Goodwin, D.L. (2017). Reflections on professional practice in adapted physical activity through a social justice lens. In A.J.S. Morin, C. Maiano, D. Tracey, \& R.G. Craven (Eds.), Inclusive physical activities: International perspectives (pp. 271-286). Information Age Publishing.

Goodwin, D.L., \& Eales, L. (2020). Trauma-informed pedagogy: Rethinking the use of graduated instructional prompts in inclusive physical education. Palaestra, 34(2), 43-48. https://js.sagamorepub.com/palaestra/article/view/10629

Goodwin, D.L., \& Rossow-Kimball, B. (2012). Thinking ethically about professional practice in adapted physical activity. Adapted Physical Activity Quarterly, 29(4), 295-309. PubMed ID: 23027144 https://doi.org/10.1123/apaq.29.4.295

Gregor, S., Bruni, N., Grkinic, P., Schwartz, L., McDonald, A., Thille, P., . . Jachyra, P. (2018). Parents' perspectives of physical activity participation among Canadian adolescents with autism spectrum disorders. Research in Autism Spectrum Disorders, 48, 53-62. https://doi.org/10.1016/j.rasd.2018.01.007

Guy-Evans, O. (2020). Bronfenbrenner's ecological systems theory. Simply Psychology. https://www.simplypsychology.org/Bronfenbrenner.html

Higgs, C. (2010). Physical literacy-Two approaches, one concept. http://www/sirc.ca/ newsletters/midhan11/documents/PhysLit_2approaches1concept.pdf

Higgs, C., Cairney, J., Jurbala, P., Dudley, D., Way, R., \& Mitchell, D. (2019). Developing physical literacy-Building a new normal for all Canadians. https://physicalliteracy.ca/wpcontent/uploads/2020/01/DPL-2_EN_web_November_2019-1.pdf

Hodge, N., \& Runswick-Cole, K. (2013). "They never pass me the ball:” Exposing ableism through the leisure experiences of disabled children, young people and their families. Children's Geographies, 11(3), 311-325. https://doi.org/10.1080/14733285.2013. 812275

Huit, W. (2012). A systems approach to the study of human behavior. Educational Psychology Interactive. http://www.edpsycinteractive.org/materials/sysmdlo.html

Jurbala, P. (2015). What is physical literacy, really? Quest, 67(4), 367-383. https://doi.org/ 10.1080/00336297.2015.1084341

Kivunja, D., \& Kuyini, A.B. (2017). Understanding and applying research paradigms in educational contexts. International Journal of Higher Education, 6(5), 26-41. https:// doi.org/10.5430/ijhe.v6n5p26

Lang, R., Koegel, L.K., Ashbaugh, K., Regester, A., Ence, W., \& Smith, W. (2010). Physical exercise and individuals with autism spectrum disorders: A systematic review. Research in Autism Spectrum Disorders, 4(4), 565-576. https://doi.org/10.1016/j.rasd. 2010.01.006

Lee, S.H., \& Hodge, S.R. (2017). Children with autism spectrum disorder and physical activity: A descriptive synthesis. Journal of Physical Education and Sport Management, 8(1), 1-23. https://doi.org/10.5897/jpesm2016.0284

Lester, J.N., Karim, K., \& O'Reilly, M. (2014). “Autism itself actually isn't a disability": Negotiating a "normal" versus "abnormal" autistic identity. Communication \& Medicine, 11(2), 13-152. https://doi.org/10.1558/cam.vlli2.20371

Lincoln, Y.S., Lynham, S.A., \& Guba, E.G. (2011). Paradigmatic controversies, contradictions, and emerging confluences. In N.K. Denzin \& Y.S. Lincoln (Eds.), Handbook of qualitative research (4th ed., pp. 97-128). Sage.

Lloyd, R.J. (2016). Becoming physically literate for life: Embracing the functions, forms, feelings and flows of alternative and mainstream physical activity. Journal of Teaching in Physical Education, 35(2), 107-116. https://doi.org/10.1123/jtpe.2015-0068 
Loprinzi, P.D., Davis, R.E., \& Fu, Y.C. (2015). Early motor skill competence as a mediator of child and adult physical activity. Preventive Medicine Reports, 2, 833-838. PubMed ID: 26844157 https://doi.org/10.1016/j.pmedr.2015.09.015

MacDonald, M., Esposito, P.E., \& Ulrich, D.A. (2011). The physical activity patterns of children with autism. BMC Research Notes, 4(1), 422-426. https://doi.org/10.1186/ 1756-0500-4-422

Markula, P., \& Silk, M. (2011). Qualitative research for physical culture. Palgrave Macmillan.

Martin, J.J., \& Choi, Y.S. (2009). Parents' physical activity-related perceptions of their children with disabilities. Disability and Health Journal, 2(1), 9-14. PubMed ID: 21122737 https://doi.org/10.1016/j.dhjo.2008.09.001

Mayan, M.J. (2009). Essentials in qualitative inquiry. Routledge.

McMahon, J., Wiltshire, G., McGannon, K.R., \& Rayner, C. (2019). Children with autism in a sport and physical activity context: A collaborative autoethnography by two parents outlining their experiences. Sport, Education and Society, 25(9), 1002-1014. https:// doi.org/10.1080/13573322.2019.1680535

Merriam, S.B., Johnson-Bailey, J., Lee, M-Y., Kee, Y., Ntseane, G., \& Muhamad, M. (2001). Power and positionality: Negotiating insider/outsider status within and across cultures. International Journal of Lifelong Education, 20(5), 405-416. https://doi.org/ $10.1080 / 02601370120490$

Milner, H.R. (2007). Race, culture, and researcher positionality: Working through dangers seen, unseen, and unforeseen. Educational Research, 36,(7) 388-400. https://journals. sagepub.com/doi/pdf/10.3102/0013189X07309471?casa_token=SSC6cQ-6njoAAAAA: W67a6jpRuhBgpCs4Jrw_Yot0P50GaFJRpWB9Oj_nWfp2se10L28dhv9x9LFlygQX fiyoxdiL7gUD9Q

Must, A., Phillips, S., Curtin, C., \& Bandini, L.G. (2015). Barriers to physical activity in children with autism spectrum disorders: Relationship to physical activity and screen time. Journal of Physical Activity and Health, 12(4), 529-534. PubMed ID: 25920014 https://doi.org/10.1123/jpah.2013-0271

O’Sullivan, M., Davids, K., Woods, C.T., Rothwell, M., \& Rudd, J. (2020). Conceptualizing physical literacy within an ecological dynamics framework. Quest, 72(4), 448-462. https://doi.org/10.1080/00336297.2020.1799828

Obrusnikova, I., \& Miccinello, D.L. (2012). Parent perceptions of factors influencing afterschool physical activity of children with autism spectrum disorders. Adapted Physical Activity Quarterly, 29(1), 63-80. PubMed ID: 22190053 https://doi.org/10.1123/apaq. 29.1.63

Ostfeld-Etzion, S., Feldman, R., Hirschler-Guttenberg, Y., Laor, N., \& Golan, O. (2016). Self-regulated compliance in preschoolers with autism spectrum disorder: The role of temperament and parental disciplinary style. Autism, 20(7), 868-878. PubMed ID: 26685197 https://doi.org/10.1177/1362361315615467

Paterson, M., \& Higgs, J. (2005). Using hermeneutics as a qualitative research approach in professional practice. The Qualitative Report, 10(2), 339-357. http://www.nova.edu/ ssss/QR/QR10-2/paterson.pdf

Patton, M.Q. (2015). Qualitative research and evaluation methods (4th ed.). Sage.

Peers, D., Spencer-Cavaliere, N., \& Eales, L. (2014). Say what you mean: Rethinking disability language in adapted physical activity quarterly. Adapted Physical Activity Quarterly, 31(3), 265-282. PubMed ID: 25028477 https://doi.org/10.1123/apaq.20130091

Pfeiffer, B., Coster, W., Snethen, G., Derstine, M., Piller, A., \& Tucker, C. (2017). Caregivers' perspectives on the sensory environment and participation in daily activities of children with autism spectrum disorder. American Journal of Occupational Therapy, 71(4), 7104220020-7104220028. https://doi.org/10.5014/ajot.2017.021360 
Phillippi, J., \& Lauderdale, J. (2018). A guide to field notes for qualitative research: Context and conversation. Qualitative Health Research, 28(3), 381-388. PubMed ID: 29298584 https://doi.org/10.1177/1049732317697102

Pitetti, K.H., Rendoff, A.D., Grover, T., \& Beets, M.W. (2007). The efficacy of a 9-month treadmill walking program on the exercise capacity and weight reduction for adolescents with severe autism. Journal of Autism and Developmental Disorders, 37(6), 997-1006. PubMed ID: 17151799 https://doi.org/10.1007/s10803-006-0238-3

Pushkarenko, K., Causgrove Dunn, J., \& Wohlers, B. (2020). Physical literacy and inclusion: A scoping review of the physical literacy literature inclusive of individuals experiencing disability. Prospects: Comparative Journal of Curriculum, Learning, and Assessment, 50, 107-126. https://doi.org/10.1007/s11125-020-09497-8

Rosa, E.M., \& Tudge, J. (2013). Urie Bronfenbrenner's theory of human development: Its evolution from ecology to bioecology. Journal of Family Theory \& Review, 5(4), 243-258. https://doi.org/10.1111/jftr.12022

Rudd, J.R., Pesce, C., Strafford, B.W., \& Davids, K. (2020). Physical literacy-A journey of individual enrichment: An ecological dynamics rationale for enhancing performance and physical activity in all. Frontiers in Psychology, 11, 1904. PubMed ID: 32849114 https://doi.org/10.3389/fpsyg.2020.01904

Shinebourne, P. (2011). Interpretative phenomenological analysis. In N. Frost (Ed.), Qualitative research methods in psychology: Combining core approaches (pp. 44-65). McGraw-Hill.

Siebert, E.A., Hamm, J., \& Yun, J. (2017). Parental influence on physical activity of children with disabilities. International Journal of Disability, Development and Education, 64(4), 378-390. https://doi.org/10.1080/1034912X.2016.1245412

Smith, B. (2018). Generalizability in qualitative research: Misunderstandings, opportunities and recommendations for the sport and exercise sciences. Qualitative Research in Sport, Exercise and Health, 10(1), 137-149. https://doi.org/10.1080/2159676X.2017. 1393221

Smith, B., \& McGannon, K.R. (2018). Developing rigor in qualitative research: Problems opportunities within sport and exercise psychology. International Review of Sport and Exercise Psychology, 11(1), 101-121. https://doi.org/10.1080/1750984X.2017. 1317357

Smith, J.A. (2017). Interpretive phenomenological analysis in sport and exercise: Getting at experience. In B. Smith \& A.C. Sparkes (Eds.). Routledge handbook of qualitative research in sport and exercise (pp. 219-229). Routledge.

Smith, J.A., Flowers, P., \& Larkin, M. (2009). Interpretive phenomenological analysis: Theory, method, and research. Sage.

Sowa, M., \& Meulenbroek, R. (2012). Effects of physical exercise on autism spectrum disorders: A meta-analysis. Research in Autism Spectrum Disorders, 6(1), 46-57. https://doi.org/10.1016/j.rasd.2011.09.001

Sparkes, A.C., \& Smith, B. (2014). Qualitative research methods in sport, exercise and health: From process to product. Routledge.

Spencer-Cavaliere, N., Thai, J., \& Kingsley, B. (2017). A part of and apart from sport: Practitioners' experiences coaching in segregated youth sport. Social Inclusion, 5(2), 120-129. https://doi.org/10.17645/si.v5i2.889

Srinivasan, S.M., Pescatello, L.S., \& Bhat, A.N. (2014). Current perspectives on physical activity and exercise recommendations for children and adolescents with autism spectrum disorders. Physical Therapy, 94(6), 875-889. PubMed ID: 24525861 https://doi.org/10.2522/ptj.20130157

Standal, O.F. (2015). Phenomenology and pedagogy in physical education. Routledge.

Stanish, H., Curtin, C., Must, A., Phillips, S., Maslin, M., \& Bandini, L. (2015). Enjoyment, barriers, and beliefs about physical activity in adolescents with and without autism 
spectrum disorder. Adapted Physical Activity Quarterly, 32(4), 302-317. PubMed ID: 26485735 https://doi.org/10.1123/APAQ.2015-0038

Tracy, S.J. (2010). Qualitative quality: Eight "big-tent" criteria for excellent qualitative research. Qualitative Inquiry, 16(10), 837-851. https://doi.org/10.1177/10778004 10383121

Tremblay, M.S., Costas-Bradstreet, C., Barnes, J.D., Bartlett, B., Dampier, D., Lalonde, C., ... Yessis, J. (2018). Canada's physical literacy consensus statement: Process and outcome. BMC Public Health, 18(Suppl. 2), 1034. https://doi.org/10.1186/s12889018-5903-X

Van Damme, T., Simons, J., Sabbe, B., \& Van West, D. (2015). Motor abilities of children and adolescents with a psychiatric condition: A systematic literature review. World Journal of Psychiatry, 5(3), 315-329. https://doi.org/10.5498/wjp.v5.i3.315

Vickerman, P., \& DePauw, K. (2010). Physical literacy and individuals with a disability. In M. Whitehead (Ed.), Physical literacy: Throughout the lifecourse (pp. 130-139). Routledge.

Whitehead, M. (2010). Physical literacy: Throughout the lifecourse. Routledge.

Whitehead, M. (2013). Definition of physical literacy and clarification of related issues. ICSSPE Bulletin, Journal of Sport Science and Physical Education, 65, 29-34. http:// www.icsspe.org/sites/default/files/bulletin65_0.pdf\#page=29

Whitehead, M. (2019). Definition of physical literacy-Development and issues. In M. Whitehead (Ed.), Physical literacy across the world (pp. 8-18). Routledge.

Whitehead, M., \& Murdoch, E. (2006). Physical literacy and physical education: Conceptual mapping. Physical Education Matters, 1(1), 6-9.

Yardley, L. (2000). Dilemmas in qualitative health research. Psychology \& Health, 15(2), 215-228. https://doi.org/10.1080/08870440008400302

Yi, K.J., Cameron, E., Patey, M., Loucks-Atkinson, A., Loeffler, T., Sullivan, A., . . Buote, R. (2020). Future directions for physical literacy: Community perspectives. Journal of Physical Education and Sport, 20(1), 123-130. https://doi.org/10.7752/jpes.2020. 01016

Zitomer, M.R., \& Goodwin, D. (2014). Gauging the quality of qualitative research in adapted physical activity. Adapted Physical Activity Quarterly, 31(3), 193-218. PubMed ID: 25028474 https://doi.org/10.1123/apaq.2013-0084 